\title{
Constraints on parity violation from ACTpol and forecasts for forthcoming CMB experiments
}

\author{
Diego Molinari ${ }^{\mathrm{a}, \mathrm{b}, *}$, Alessandro Gruppuso ${ }^{\mathrm{b}, \mathrm{c}}$, Paolo Natoli $^{\mathrm{a}}$ \\ ${ }^{a}$ Dipartimento di Fisica e Scienze della Terra, Università degli Studi di Ferrara, and INFN, \\ Sezione di Ferrara, Via Saragat 1, I-44122 Ferrara, Italy \\ ${ }^{b}$ INAF-IASF Bologna, Istituto di Astrofisica Spaziale e Fisica Cosmica di Bologna, Istituto \\ Nazionale di Astrofisica, via Gobetti 101, I-40129 Bologna, Italy \\ ${ }^{c}$ INFN, Sezione di Bologna, Via Irnerio 46, I-40126 Bologna, Italy
}

\begin{abstract}
We use the ACTpol published cosmic microwave background (CMB) polarization data to constrain cosmological birefringence, a tracer of parity violation beyond the standard model of particle physics. To this purpose, we employ all the polarized ACTpol spectra, including the cross-correlations between temperature anisotropy and B mode polarization (TB) and between E mode and $B$ mode (EB), which are most sensitive to the effect. We build specific, socalled D-estimators for birefringence and assess their performances and error budgets by using realistic Monte Carlo simulations based on the experimental characteristics provided by the ACTpol collaboration. We determine the optimal multipole range for our analysis to be $250<\ell<3025$ over which we find a null result for the uniform birefringence angle $\alpha=0.29^{\circ} \pm 0.28^{\circ}$ (stat.) $\pm 0.5^{\circ}$ (syst.), the latter uncertainty being the estimate published by the ACTpol team on their global systematic error budget. We show that this result holds consistently when other multipole ranges are considered. Finally, we forecast the capability of several forthcoming ground based, balloon and space borne CMB experiments to constrain the birefringence angle, showing, e.g., that the proposed post-Planck COrE satellite mission could in principle constrain $\alpha$ at a level of 10 arcsec, provided that all systematics are under control. Under the
\end{abstract}

\footnotetext{
* Corresponding author

Email address: molinari@iasfbo.inaf.it (Diego Molinari)
}

Preprint submitted to Journal of ${ }^{A} T_{E} X$ Templates

October 15, 2018 
same circumstances, we find the COrE constraints to be at least 2 or 3 times better than what could ideally be achieved by the other experiments considered. Keywords: CMB polarization, Fundamental physics, data analysis, CMB observations

2010 MSC: 00-01, 99-00

\section{Introduction}

Observations of the CMB radiation from third generation experimental efforts such as the Planck [1] satellite and the ground based BICEP 2/Keck [2], ACT [3] and SPT [4] observatories have ushered a new era in precision cos5 mology. Measurements of the temperature anisotropy pattern has reached very high signal-to-noise ratios and are basically limited by the sky either because of cosmic variance or because of residual errors from small scale foregrounds [5]. The experiments consider a wide range of angular scales depending on the instrument, i.e. $2 \leq \ell \lesssim 2500$ for Planck [5], $300 \lesssim \ell \lesssim 8500$ for ACT [6], $2000 \lesssim \ell \lesssim 13000$ for SPT [7. They have also released the first CMB polarization measurements with significantly high signal-to-noise ratios to effectively exploit cosmological information in the polarized components of the CMB. New observational campaigns from ground based experiments (BICEP 3 [8, Advanced ACT [9], SPTpol [10]), balloon-borne experiments (LSPE 11]) and satellite proposals (LiteBIRD [12, COrE [13]) are moving towards cosmic variance limited measurements also in polarization. This effort is motivated, other than by the will to better constrain the cosmological standard model, also by the unique possibility offered from CMB polarization to test for new physics.

Cosmic birefringence, the in vacuo rotation of the photons polarization direction during propagation [14], is already considered as a standard tracer of parity violating mechanisms beyond the Maxwell Lagrangian. Several theoretical models exhibit such an effect including Chern-Simons type interactions in the electromagnetic Lagrangian [14, a quintessence field [15, 16, axion-like particles coupled with the electromagnetic field [17], spatial anisotropies during 
evolution of perturbations [18. No detection of in vacuo birefringence has been claimed today: the effect has been constrained by laboratory experiments to be small [19]. Astrophysical probes are very good candidates for precision measurements because of the long journey engaged by cosmological photons. Distant radio galaxies are widely studied to test for parity violations (see [15, 20, 21] 30 and references therein).

Cosmic birefringence may also affect CMB photons, causing a non-zero crosscorrelation between temperature anisotropies and curl-like polarization patterns or B modes, and between gradient-like polarization patterns, or E modes, and B modes. These correlations can be parametrized by an angle $\alpha$ known as the ${ }_{35}$ birefringence angle 1 In this paper we restrict to the case of a uniform rotation angle $\alpha$, whose effect on the CMB spectra is [22, 23]:

$$
\begin{aligned}
C_{\ell}^{T E, o b s} & =C_{\ell}^{T E} \cos (2 \alpha) \\
C_{\ell}^{T B, o b s} & =C_{\ell}^{T E} \sin (2 \alpha) \\
C_{\ell}^{E E, o b s} & =C_{\ell}^{E E} \cos ^{2}(2 \alpha)+C_{\ell}^{B B} \sin ^{2}(2 \alpha) \\
C_{\ell}^{B B, o b s} & =C_{\ell}^{B B} \cos ^{2}(2 \alpha)+C_{\ell}^{E E} \sin ^{2}(2 \alpha) \\
C_{\ell}^{E B, o b s} & =\frac{1}{2}\left(C_{\ell}^{E E}-C_{\ell}^{B B}\right) \sin (4 \alpha)
\end{aligned}
$$

where $C_{\ell}^{X Y, o b s}$ and $C_{\ell}^{X Y}$ are the observed and the primordial (i.e. unrotated) angular power spectra (APS) of the $X Y$ spectrum $(X, Y=T, E$ or $B)$ respectively. The most constraining CMB spectra are TB and EB that are predicted

40 to be zero by the standard cosmological model [22]. Existing limits on $\alpha$ are all compatible with a null effect, for a review see [24, 25]. Recent analyses of uniform rotation angle obtained with Planck data can be found in [26, 27]. For anisotropic birefringence angle constraints see [28, 29]. Studies of the interplay between primordial B modes and birefringence angles are e.g. given in [30, 31.

In this paper we provide constraints of the birefringence angle employing an

\footnotetext{
${ }^{1}$ We use the customary convention used by the CMB community for the $\mathrm{Q}$ and U Stokes parameters, see e.g. http://wiki.cosmos.esa.int/planckpla2015/index.php/Sky_temperature_maps
} 
independent analysis of the polarization data recently published by the ACTpol Collaboration [9]. Moreover we give forecasts of $\alpha$ for future CMB experiments. The structure of this paper is the following: in Section 2 we present the method employed to estimate the birefringence angle; in Section 3 we describe the details of the data analysis and the procedure followed to generate realistic Monte Carlo (MC) simulations. We present our results from the ACTpol analyses in Section 4 whilst in Section 5 we provide our forecasts for several forthcoming and proposed CMB experiments. We draw our main conclusions in Section 6 .

\section{Birefringence estimators}

To find an estimate, $\tilde{\alpha}$, for the birefringence angle, $\alpha$, we consider the socalled D-estimators (see [32, 33, 34, 35, 36, 37, for details) defined as:

$$
\begin{aligned}
& D_{\ell}^{T B}(\tilde{\alpha})=C_{\ell}^{T B, o b s} \cos (2 \tilde{\alpha})-C_{\ell}^{T E, o b s} \sin (2 \tilde{\alpha}), \\
& D_{\ell}^{E B}(\tilde{\alpha})=C_{\ell}^{E B, o b s} \cos (4 \tilde{\alpha})-\frac{1}{2}\left(C_{\ell}^{E E, o b s}-C_{\ell}^{B B, o b s}\right) \sin (4 \tilde{\alpha}) .
\end{aligned}
$$

When $\tilde{\alpha}=\alpha$ the expectation value of Eq. (6) and Eq. (7) is zero. This can be easily seen by replacing Eq. (2)-(5) in Eq. (6), (7) [37.

In order to find $\tilde{\alpha}$, we minimize the following figure of merit:

$$
\chi_{X}^{2}(\tilde{\alpha})=\sum_{\ell \ell^{\prime}} D_{\ell}^{X} M_{\ell \ell^{\prime}}^{X X^{-1}} D_{\ell^{\prime}}^{X}
$$

where $X$ stands for either TB or EB and the matrix $M_{\ell \ell^{\prime}}^{X X}=\left\langle D_{\ell}^{X} D_{\ell^{\prime}}^{X}\right\rangle$ is

60 the covariance matrix of the considered D-estimator. Instead of using Eq. 8 separately for either of the D-estimators, one may consider a joint figure of merit, $D_{T B E B}$, for the vector $\left(D_{\ell}^{T B}, D_{\ell}^{E B}\right)$ that should take into account also the correlations between TB and EB spectra [37.

The analysis can be also performed after having divided the entire multipole range in small intervals, and then by minimizing the figure of merit for each interval. This probes the dependency of $\tilde{\alpha}$ from the angular scale:

$$
\chi_{X, \ell}^{2}(\tilde{\alpha})=\sum_{\ell^{\prime}} D_{\ell}^{X} M_{\ell \ell^{\prime}}^{X X^{-1}} D_{\ell^{\prime}}^{X} .
$$

Resumming over $\ell$ we obtain again Eq. (8). 


\section{Data and simulations}

We analyse the recent data coming from the first three months of ACTpol observations covering 270 square degrees at $146 \mathrm{GHz}$ with 1.3 arcminute resolution FWHM. These data are described in 9] and the 6 CMB APS are publicly available $2^{2}$. They cover the multipole range $225 \leq \ell \leq 8725$ in bins varying from $\Delta \ell=50$ for $225 \leq \ell \leq 2025$ to $\Delta \ell=800$ for $6325 \leq \ell \leq 8725$. In Fig. 1 we show the ACTpol measurements compared to the Planck best fit $\Lambda$ CDM model [39].

In order to propagate the errors through our analysis, we generate a realistic MC of 10000 simulated CMB APS based on the Planck 2015 ACDM best fit model. We work directly in harmonic space; to generate a set of 6 correlated CMB APS we build, for each bin, $\hat{\ell}$, a $6 \times 6$ covariance matrix, $A_{\hat{\ell}}$ :

$$
A_{\hat{\ell}}=\left(\begin{array}{cccccc}
\left(\sigma_{\hat{\ell}}^{T T}\right)^{2} & \Delta_{\hat{\ell}}^{T T E E} & 0 & \Delta_{\hat{\ell}}^{T T T E} & 0 & 0 \\
\Delta_{\hat{\ell}}^{T T E E} & \left(\sigma_{\hat{\ell}}^{E E}\right)^{2} & 0 & \Delta_{\hat{\ell}}^{E E T E} & 0 & 0 \\
0 & 0 & \left(\sigma_{\hat{\ell}}^{B B}\right)^{2} & 0 & 0 & 0 \\
\Delta_{\hat{\ell}}^{T T T E} & \Delta_{\hat{\ell}}^{E E T E} & 0 & \left(\sigma_{\hat{\ell}}^{T E}\right)^{2} & 0 & 0 \\
0 & 0 & 0 & 0 & \left(\sigma_{\hat{\ell}}^{T B}\right)^{2} & \Delta_{\hat{\ell}}^{T B E B} \\
0 & 0 & 0 & 0 & \Delta_{\hat{\ell}}^{T B E B} & \left(\sigma_{\hat{\ell}}^{E B}\right)^{2}
\end{array}\right)
$$

where we use as diagonal elements the published ACTpol variances, $\left(\sigma_{\hat{\ell}}^{X}\right)^{2},(X=$ $T T, E E, B B, T E, T B$ or $E B)$. Since we consider also primordial BB spectrum, 75 in addition to the correlations between the spectra $\mathrm{TT}$ and $\mathrm{EE}\left(\Delta_{\hat{\ell}}^{T T E E}\right)$, TT and TE, $\left(\Delta_{\hat{\ell}}^{T T T E}\right)$, and EE and TE, $\left(\Delta_{\hat{\ell}}^{E E T E}\right)$ as considered in [40, 41], we take into account also correlations between TB and EB, $\left(\Delta_{\hat{\ell}}^{T B E B}\right)$. Assuming the diagonal elements as given, the non-zero off diagonal terms of the $A_{\hat{\ell}}$ matrix

\footnotetext{
${ }^{2}$ Available at http://lambda.gsfc.nasa.gov/product/act/actpol_prod_table.cfm . Note that
} the ACTpol published power spectra bands have not been subject to a nulling technique to ensure that TB and EB are compatible with zero. The technique is only used to assess the systematic error in polarization orientation 38 . 

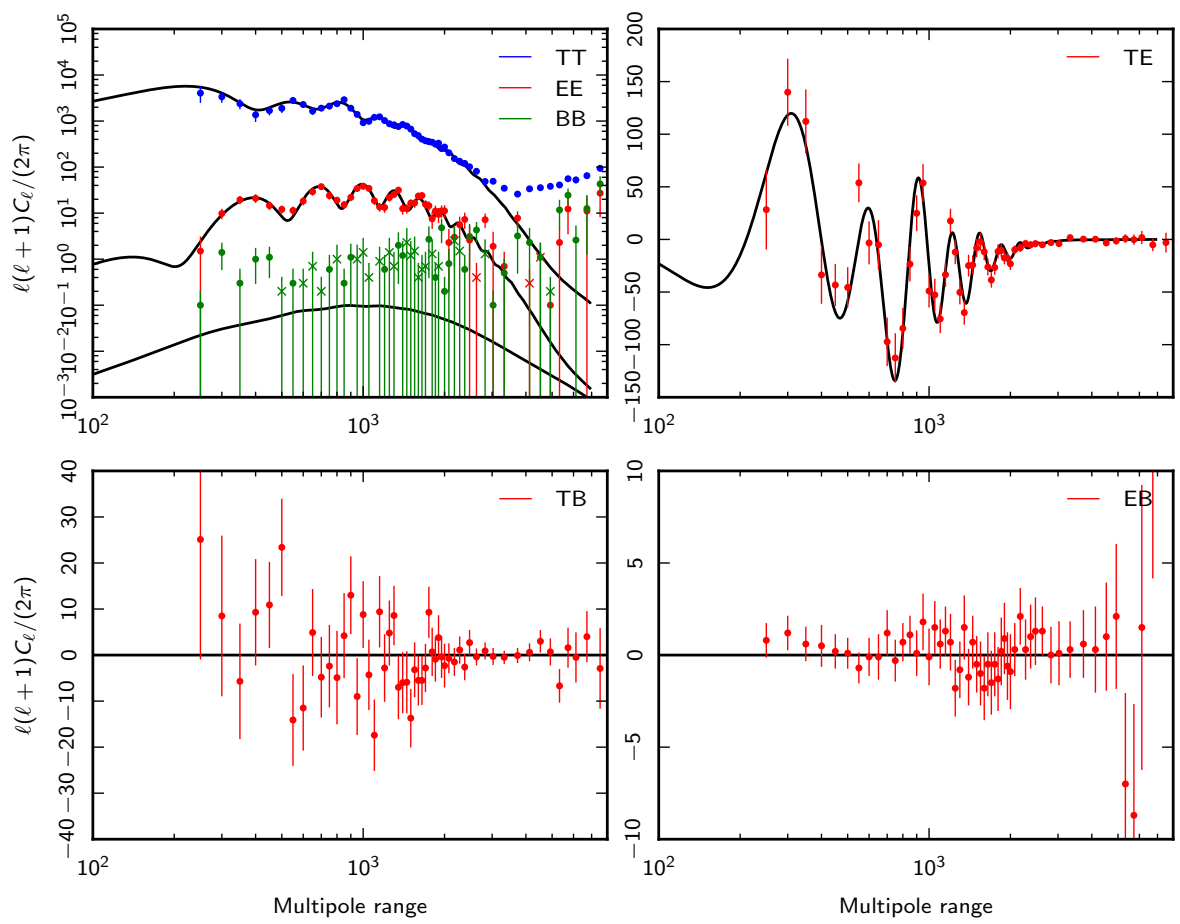

Figure 1: ACTpol 2014 spectra described in [9]. Spectra are as labelled. The black curve is the Planck best fit $\Lambda$ CDM model. Notice the marked deviation from model in the TT APS for $\ell \gtrsim 3000$. This is due to contamination from small scales foregrounds. It has no effect on our analysis since we do not consider TT. A possible bias in EE at small scales has been constrained in 9] to be below $3 \sigma$, putting a limit on the amplitude of polarized sources. We ignore this effect hereafter. The ' $\mathrm{x}$ ' symbols for $\mathrm{BB}$ and $\mathrm{EE}$ flag points that happen to be negative and for which the absolute value is displayed.

can be written as:

$$
\begin{aligned}
\Delta_{\hat{\ell}}^{T T E E} & =\frac{2}{(2 \hat{\ell}+1) f_{s k y}}\left(C_{\hat{\ell}}^{T E}\right)^{2} \\
\Delta_{\hat{\ell}}^{T T T E} & =\sqrt{\frac{2}{(2 \hat{\ell}+1) f_{s k y}}}\left(C_{\hat{\ell}}^{T E} \sigma_{\hat{\ell}}^{T T}\right) \\
\Delta_{\hat{\ell}}^{E E T E} & =\sqrt{\frac{2}{(2 \hat{\ell}+1) f_{s k y}}}\left(C_{\hat{\ell}}^{T E} \sigma_{\hat{\ell}}^{E E}\right) \\
\Delta_{\hat{\ell}}^{T B E B} & =\sqrt{\frac{1}{2(2 \hat{\ell}+1) f_{s k y}}}\left(C_{\hat{\ell}}^{T E} \sigma_{\hat{\ell}}^{B B}\right)
\end{aligned}
$$


where $C_{\hat{\ell}}^{T E}$ is taken from the Planck $2015 \Lambda \mathrm{CDM}$ best fit model and $f_{\text {sky }}$ is the effective sky fraction used for the APS estimation. While this value is not quoted by the ACTpol team, it can be inferred from the magnitude of the published error bars. In any case, we find that the impact of the off-diagonal elements of the covariance matrix is weak. We also assume that the noise does not correlate among temperature and polarization. Note that the above procedure allows one to account not only for white noise and cosmic variance, but also for other (systematic) effects that are already present in the published error bars of the spectra of ACTpol data 9 .

To build a MC set of simulated spectra, we use the Cholesky factor of Equation 10 assuming a reference $\Lambda \mathrm{CDM}$ model. We then compare the estimates $\tilde{\alpha}$ from the MC simulations with those from real data.

In Fig. 2 we show the mean and standard deviation of the $10000 \mathrm{MC}$ simulated APS.

\section{Results}

We compute and minimize the figure of merit of Eq. (8) for the ACTpol data and the MC simulations, considering the multipole range [250,3025]. The minimum multipole is dictated by the ACTpol data, while the choice of the maximum multipole is derived by considerations of the signal-to-noise ratio, highlighted in Appendix A.

In Fig. 3 we show the empirical distribution of the estimated birefringence angle for $D_{T B}$ (left panel), $D_{E B}$ (central panel) and $D_{T B E B}$ (right panel) of the MC. The dark blue bar highlights the value derived from the ACTpol data. In Fig. 4 we show the results obtained using the $D_{T B}$ (blue bars), $D_{E B}$ (red bars) or $D_{T B E B}$ (green bars) estimators, considering several multipole ranges.

In addition to the range $[250,3025]$ we also take into account the multipole interval $[250,6125]$ which comprises the entire set of useful power spectra bands provided by the ACTpol. Moreover we consider the multipole range [250, 2000] for consistency test. All our constraints are well compatible each other as re- 

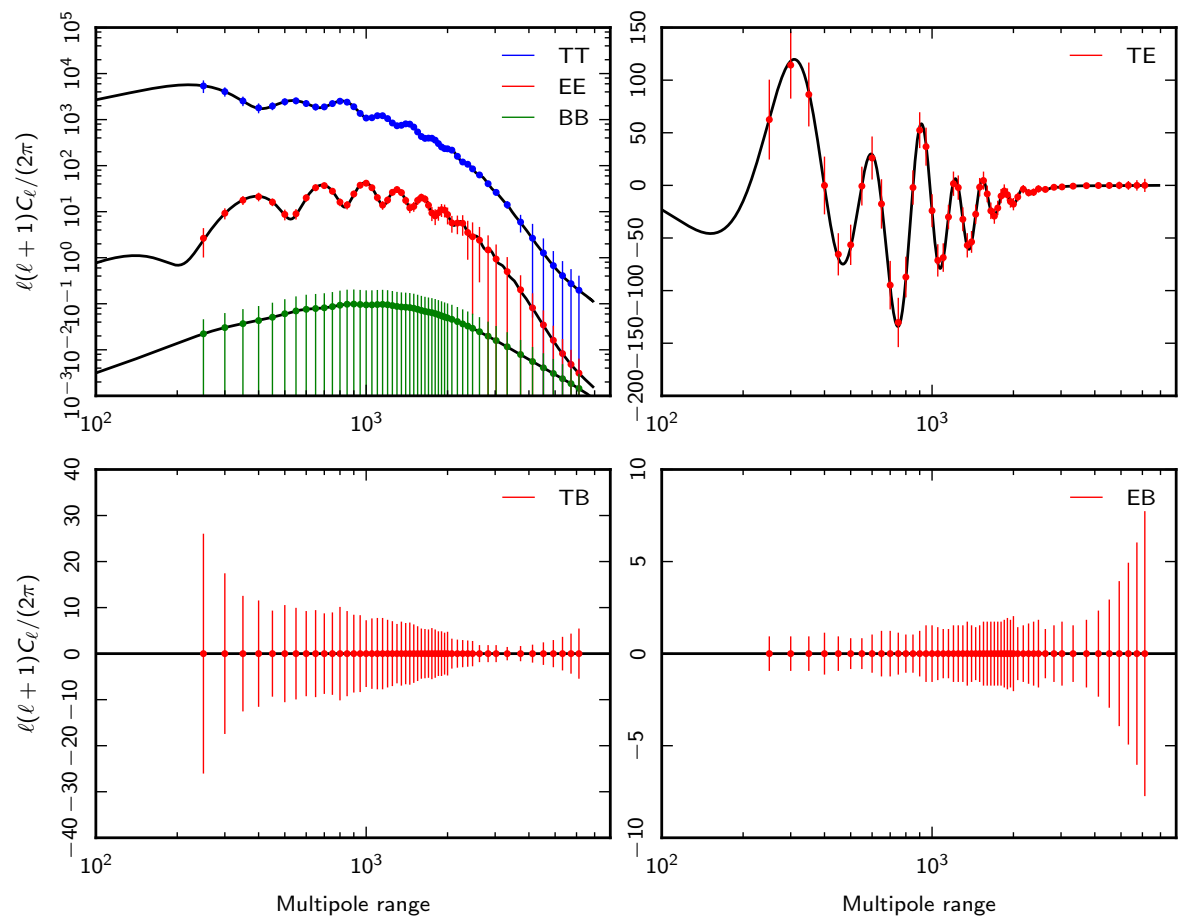

Figure 2: Average and dispersion of the 10000 simulated APS. Clockwisely the TT, EE and BB APS (top left panel), the TE APS (top right panel), the EB APS (bottom right panel) and the TB APS (bottom left panel).

\begin{tabular}{|c|c|c|c|}
\hline Multipole range & $D_{T B}[\mathrm{deg}]$ & $D_{E B}[\mathrm{deg}]$ & $D_{T B E B}[\mathrm{deg}]$ \\
\hline $250-6125$ & $0.32 \pm 0.82$ & $0.34 \pm 0.29$ & $0.29 \pm 0.28$ \\
\hline $250-3025$ & $0.40 \pm 0.82$ & $0.32 \pm 0.29$ & $0.29 \pm 0.28$ \\
\hline $250-2000$ & $0.33 \pm 0.83$ & $0.31 \pm 0.30$ & $0.28 \pm 0.28$ \\
\hline
\end{tabular}

Table 1: Estimated birefringence angle using $D_{T B}, D_{E B}$ or $D_{T B E B}$ for several multipole ranges (first column). Note the overall consistency of the estimated angles. 


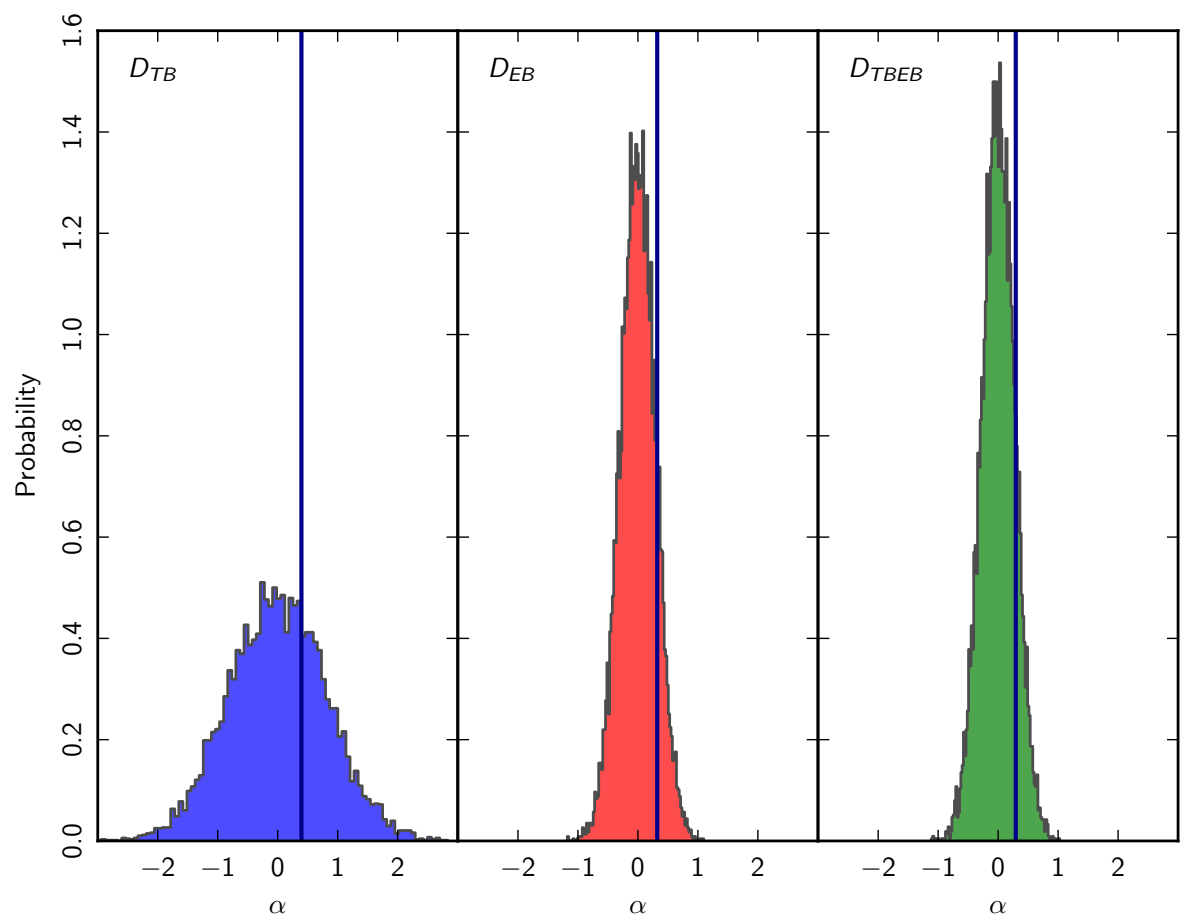

Figure 3: Normalized distributions of birefringence angles estimated from the MC simulations using $D_{T B}$ (left panel), $D_{E B}$ (central panel) and $D_{T B E B}$ (right panel) compared with the ones derived from the ACTpol data (dark blue). 


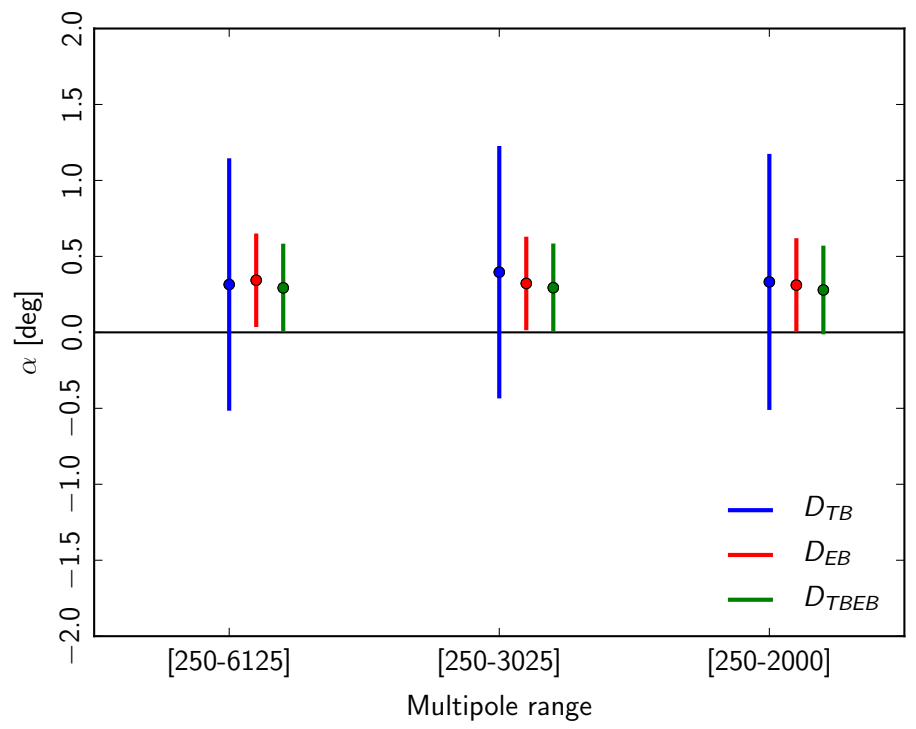

Figure 4: Estimates of the birefringence angle from $D_{T B}$ (blue bars), $D_{E B}$ (red bars) or $D_{T B E B}$ (green bars) for several multipole ranges.

ported in Table 1. We also addressed the impact of the cross-correlations between TB and EB spectra in the $D_{T B E B}$ estimator finding it negligible.

Our best, representative estimate is $\tilde{\alpha}=(0.29 \pm 0.28)^{\circ}$ (all the statistical errors given in this paper are at $1 \sigma$ level). It is in good agreement with previous analyses [42, 36] showing an estimated birefringence angle consistent with zero at about $1 \sigma$. The error quoted above is purely statistical and does not include a systematic contribution estimated in $\left[9\right.$ at $0.5^{\circ}$. This error is of the same order of magnitude as the statistical error derived above so both have to be considered together when quoting the ACTpol constraints on $\alpha$.

\subsection{Consistency tests across multipole ranges}

The D-estimators can be used to extract $\tilde{\alpha}$ in different multipole ranges as in Eq. (9). In Fig. 5 we show estimates of the uniform rotation angle $\tilde{\alpha}$ as obtained at different angular scales by dividing the multipole range $[250,3025]$ in 9 intervals, i.e. in bins of $\Delta \ell=250$ up to $\ell=1950, \Delta \ell=400$ up to $\ell=2375$ and $\Delta \ell=600$ up to $\ell=3025$. Errors blow up significantly beyond 


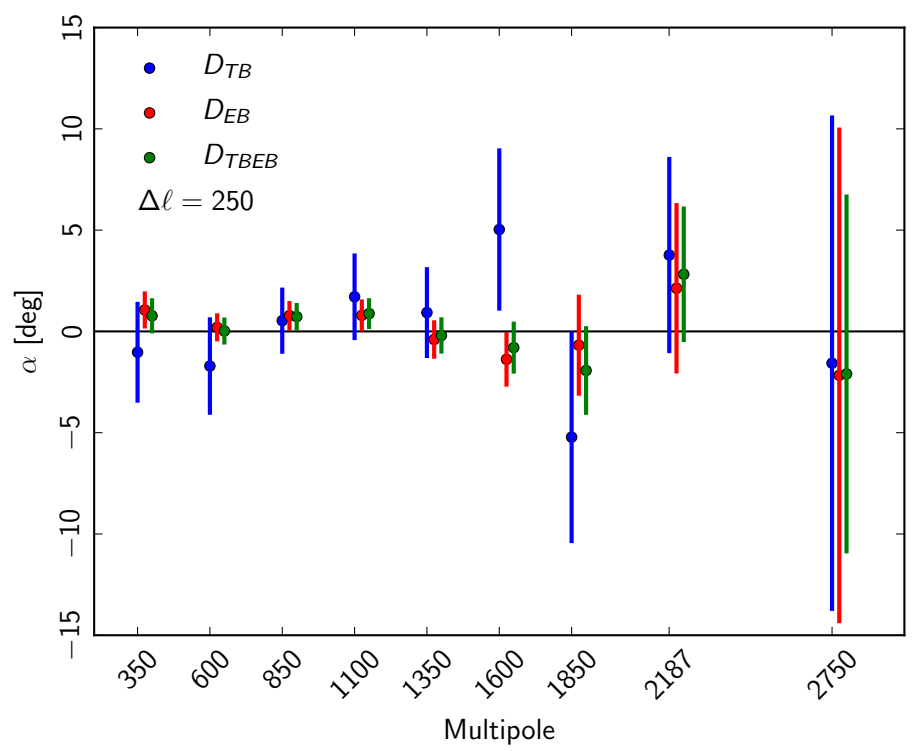

Figure 5: Extraction of the uniform birefringence angle considering the multipole range $[250,3025]$ divided in 9 subintervals with $\Delta \ell=250$ up to $\ell=1950, \Delta \ell=400$ up to $\ell=2375$ and $\Delta \ell=600$ up to $\ell=3025$.

this value. We find that all the estimates of the uniform birefringence angle do not significantly deviate from zero in any subinterval considered. This shows that the assumption of a uniform rotation angle continues to hold also when subsets of the full multipole range are considered.

\section{Forecasts for future experiments}

In this Section we derive forecasts for forthcoming space-born and sub-orbital experiments using the machinery described above.

\subsection{Methodology}

We build the covariance matrix by considering the explicit covariances for an idealized white noise only experiment, 40, 41, with gaussian circular beam. We allow for the possibility of having non zero primordial B modes, but assume that the primordial TB and EB spectra are zero, i.e. there is no parity violating 


\begin{tabular}{|c|c|c|c|c|c|}
\hline Description & $\nu$ & $f_{\text {sky }}$ & Beam & $\sigma_{T}$ & $\sigma_{P}$ \\
\hline & {$[\mathrm{GHz}]$} & {$[\%]$} & {$[\operatorname{arcmin}]$} & {$[\mu K$-arcmin $]$} & {$[\mu K$-arcmin $]$} \\
\hline Advanced ACT[6] & 150 & 50 & 1.4 & 7.0 & 9.9 \\
\hline LSPE[1] & 95 & 20 & 110 & 7.8 & 11.0 \\
\hline LiteBIRD[12] & 140 & 80 & 32 & 3.7 & 5.2 \\
\hline COrE-like sat.[13] & 135 & 80 & 7.8 & 2.63 & 4.55 \\
\hline Ideal (r=0.1) & - & 100 & - & - & - \\
\hline Ideal (r=0.01) & - & 100 & - & - & - \\
\hline
\end{tabular}

Table 2: List of the forecasts considered. We consider only the frequency with most favourable sensitivity to $\mathrm{CMB}$ polarization. For the definition of temperature and polarization sensitivities see subsection 5.1

signal from the early universe. The diagonal elements of the covariance matrix read:

$$
\begin{aligned}
& \left(\Delta C_{\ell}^{T T}\right)^{2}=\frac{2}{(2 \ell+1) f_{s k y}}\left(C_{\ell}^{T T}+w_{T}^{-1} W_{\ell}^{-2}\right)^{2} \\
& \left(\Delta C_{\ell}^{E E}\right)^{2}=\frac{2}{(2 \ell+1) f_{s k y}}\left(C_{\ell}^{E E}+w_{P}^{-1} W_{\ell}^{-2}\right)^{2} \\
& \left(\Delta C_{\ell}^{B B}\right)^{2}=\frac{2}{(2 \ell+1) f_{s k y}}\left(C_{\ell}^{B B}+w_{P}^{-1} W_{\ell}^{-2}\right)^{2} \\
& \left(\Delta C_{\ell}^{T E}\right)^{2}=\frac{1}{(2 \ell+1) f_{s k y}}\left[\left(C_{\ell}^{T T}+w_{T}^{-1} W_{\ell}^{-2}\right)\left(C_{\ell}^{E E}+w_{P}^{-1} W_{\ell}^{-2}\right)+\left(C_{\ell}^{T E}\right)^{2}\right] \\
& \left(\Delta C_{\ell}^{T B}\right)^{2}=\frac{1}{(2 \ell+1) f_{s k y}}\left[\left(C_{\ell}^{T T}+w_{T}^{-1} W_{\ell}^{-2}\right)\left(C_{\ell}^{B B}+w_{P}^{-1} W_{\ell}^{-2}\right)\right] \\
& \left(\Delta C_{\ell}^{E B}\right)^{2}=\frac{1}{(2 \ell+1) f_{s k y}}\left[\left(C_{\ell}^{E E}+w_{T}^{-1} W_{\ell}^{-2}\right)\left(C_{\ell}^{B B}+w_{P}^{-1} W_{\ell}^{-2}\right)\right]
\end{aligned}
$$

where $f_{s k y}$ is the useful sky fraction available, $W_{\ell}$ is a (Gaussian) window function, $w_{T, P}^{-1}=\sigma_{T, P}^{2}$ are the inverse statistical weights per unit solid angle and $\sigma_{T, P}$ are the noise sensitivities per unit solid angle of the instrument in temper- 
ature and polarization. The non-zero off diagonal terms are:

$$
\begin{aligned}
\operatorname{Cov}\left(C_{\ell}^{T T}, C_{\ell}^{E E}\right) & =\frac{2}{(2 \ell+1) f_{s k y}}\left(C_{\ell}^{T E}\right)^{2} \\
\operatorname{Cov}\left(C_{\ell}^{T T}, C_{\ell}^{T E}\right) & =\frac{2}{(2 \ell+1) f_{s k y}}\left[C_{\ell}^{T E}\left(C_{\ell}^{T T}+w_{T}^{-1} W_{\ell}^{-2}\right)\right] \\
\operatorname{Cov}\left(C_{\ell}^{E E}, C_{\ell}^{T E}\right) & =\frac{2}{(2 \ell+1) f_{s k y}}\left[C_{\ell}^{T E}\left(C_{\ell}^{E E}+w_{P}^{-1} W_{\ell}^{-2}\right)\right] \\
\operatorname{Cov}\left(C_{\ell}^{T B}, C_{\ell}^{E B}\right) & =\frac{1}{(2 \ell+1) f_{s k y}}\left[C_{\ell}^{T E}\left(C_{\ell}^{B B}+w_{P}^{-1} W_{\ell}^{-2}\right)\right]
\end{aligned}
$$

\subsection{Experimental set-up}

In Table 2 we show the experiments we consider and their main features. All of them are multi-frequency instruments. As reference, we consider the single frequency with the most favourable sensitivity to CMB polarization. The new observational campaign of the ACT telescope, called Advanced ACT [6], will observe about $50 \%$ of the sky for 3 years. At $150 \mathrm{GHz}$ it will feature a beam of 1.4 arcmin reaching a noise level of $7 \mu \mathrm{K}$-arcmin in temperature and $9.9 \mu K$-arcmin in polarization. The balloon experiment LSPE [11] will observe $20 \%$ of the sky between $30 \mathrm{GHz}$ and $245 \mathrm{GHz}$ with a telescope beam of 110 arcmin at $95 \mathrm{GHz}$ reaching a map noise level of $7.8 \mu \mathrm{K}$-arcmin in temperature and $11.0 \mu K$-arcmin in polarization. The LiteBIRD satellite[12] will observe the CMB sky between $60 \mathrm{GHz}$ and $280 \mathrm{GHz}$ with a beam of 32 arcmin at 145 $\mathrm{GHz}$ and a sensitivity of $3.7 \mu K$-arcmin in temperature and $5.2 \mu K$-arcmin in polarization. A COrE-like satellite 13 may observe the CMB sky between 45 $\mathrm{GHz}$ ad $795 \mathrm{GHz}$ with a resolution of 7.8 arcmin at $135 \mathrm{GHz}$ and a noise level of $2.63 \mu K$-arcmin in temperature and $4.55 \mu K$-arcmin in polarization. For these last two cases we consider $f_{s k y}=80 \%$, a reasonable value allowing for some expected developments in the component separation techniques. Since the sky fraction available for $\mathrm{CMB}$ analysis is expected to be large for these future experiments, we consider a multipole range that differs in extension and binning size from the one employed above for ACTpol. We thus consider $\Delta \ell=10$ up to $\ell=500, \Delta \ell=50$ up to $\ell=2000, \Delta \ell=100$ up to $\ell=2500$ and $\Delta \ell=200$ up to $\ell=3000$. For the LiteBIRD and CORE we consider $\ell_{\text {min }}=2$ since 
they will analyse a larger portion of the sky, for Advanced ACT and LSPE perfectly deconvolved. Thus, for the following analysis, we consider MC of unlensed CMB APS. The impact on $\alpha$ of a misestimation of the lensing kernel is addressed in 37. 


\subsection{Results}

We compute the figure of merit of Eq. (8) using a MC of 10000 unlensed CMB APS. We report in Fig. 6 the expected $1 \sigma$ error on the estimate of the birefringence angle $\tilde{\alpha}$ for each experiment considered in Table 2, The ideal full sky cases can be interpreted as the cosmic variance limit of our D-estimators assuming two different fiducial values for $r$.

The best prospects for constraining $\alpha$ are provided by a COrE-like satellite mission which will be able to achieve a sensitivity for $\alpha$ of the level of 10 arcsec. This of course assume negligible impact of systematic effects from both the sky and the instrument. Under the same condition the LiteBIRD mission has a sensitivity 4 times coarser, due to its poorer resolution, which limits the multipole range available for the analysis. The higher resolution ground based Advanced ACT experiment achieves a sensitivity on $\alpha$ which is intermediate between LiteBIRD and COrE. Despite its coarser angular resolution the LSPE balloon borne experiment can achieve bounds on $\alpha$ about twice as stringent as present day high resolution experiments. The ideal full sky cases show that cosmic variance is still far from being the dominant source of error for future experiments: even for COrE noise represents the dominant source of uncertainty (again ignoring systematic effects) being at least two order of magnitude larger than cosmic variance. In fact, cosmic variance alone prevents detection of $\alpha$ to better than 0.02 arcsec for $r=0.01$. This limit becomes even smaller assuming lower values of the tensor-to-scalar ratio.

For the case of COrE we have checked the impact of halving the accessible multipole range of the mission by setting $\ell_{\max }=1000$, finding a small impact on the error estimate of $\alpha$ (less than 10\%). In fact the highest leverage to the constraining power in $\alpha$ comes from the multipole region below $\ell=1500$.

\section{Conclusions}

We have applied the well known D-estimators for the birefringence angle to the recently published $\mathrm{ACT}$ pol data using a frequentist $\mathrm{MC}$ based approach. 


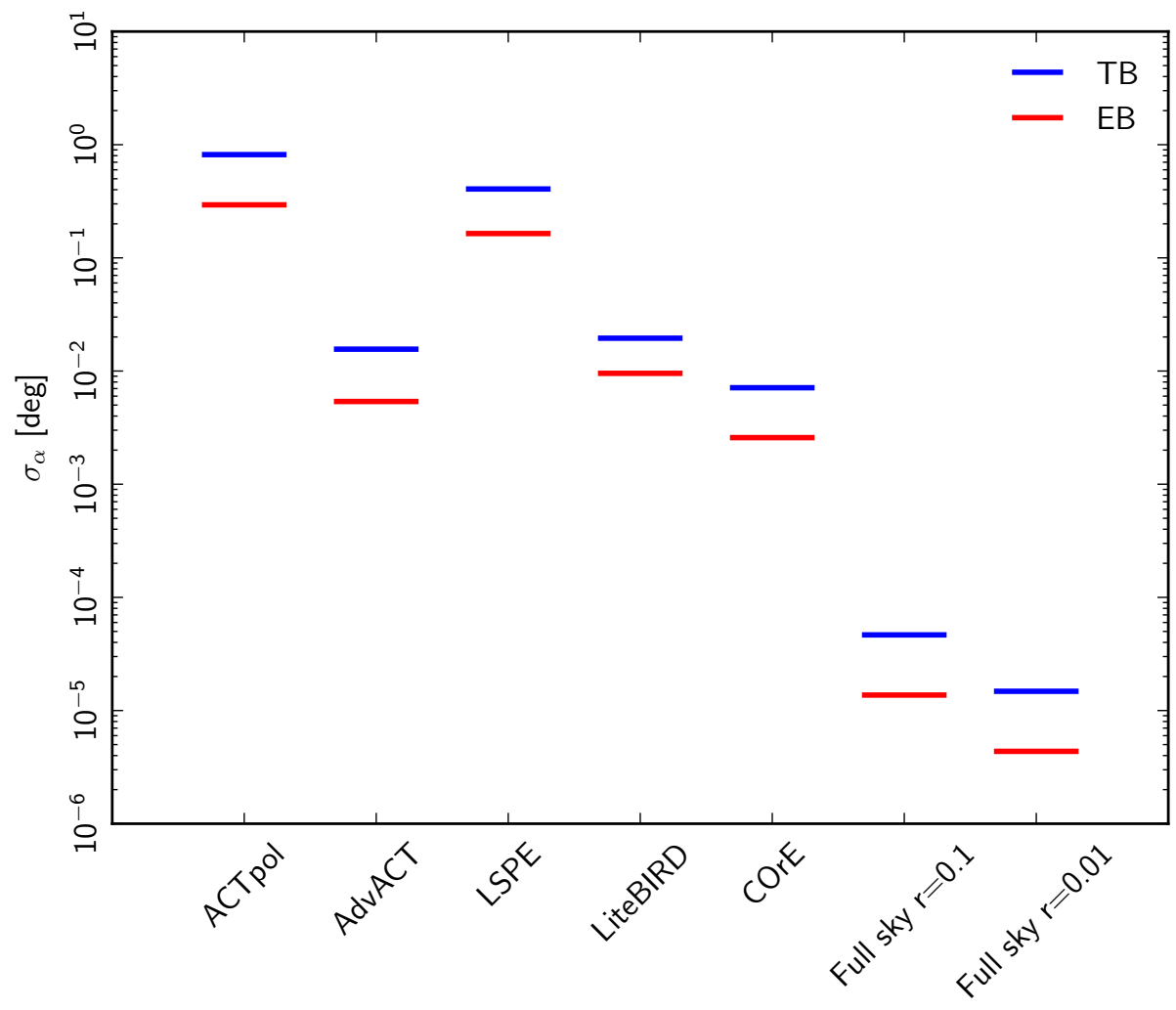

Figure 6: Forecasts for $1 \sigma$ errors in the measurements of the birefringence angle using the D-estimators, $D_{T B}$ (blue) and $D_{E B}$ (red) for the future experiments listed. We also quote the error estimates achievable with public ACTpol data discussed above in this paper and two ideal, cosmic variance limited, experiments assuming a value of the tensor-to-scalar ratio $r=0.1$ or 0.01 respectively. We do not show the $D_{E B T B}$ results because they are in practice undistinguishable from the $D_{E B}$ ones. 

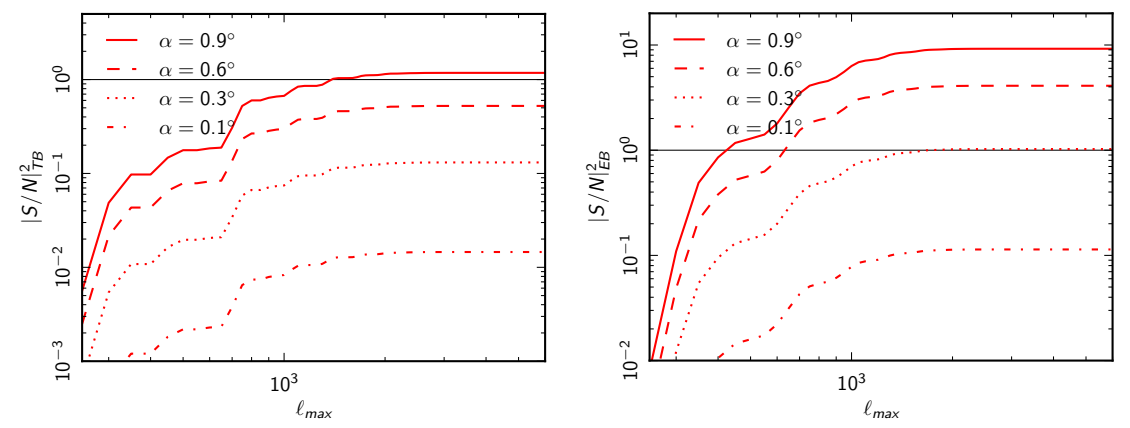

Figure 7: Left panel: Signal-to-noise ratio for TB as defined in Eq. 25 for ACTpol data and considering several $\alpha=0.9^{\circ}$ (solid), $\alpha=0.6^{\circ}$ (dashed), $\alpha=0.3^{\circ}$ (dotted), $\alpha=0.1^{\circ}$ (dash dot). Right panel: As in the left panel but for EB as defined in Eq. 26.

Our best estimate for the uniform birefringence angle is $\tilde{\alpha}=0.29^{\circ} \pm 0.28^{\circ}$ (stat.) $\pm 0.5^{\circ}$ (syst.) compatible with no detection and in agreement with previous analyses [42, 36]. The systematic error is an estimate given by the ACTpol collaboration 9 .

We have also presented the dependence of uniform $\alpha$ on subintervals of the whole multipole range used for the global analysis. This test that was not previously known for ACTpol, provides no significant deviation from zero in any of the subintervals considered.

Furthermore, we used our MC machinery to provide forecasts for the planned experiments Advanced ACT, LSPE, LiteBIRD and as well as a possible COrElike mission. We find that the best sensitivity is achieved by COrE that may be able to constrain the birefringence angles at a level of 10 arcsec provided that all systematic effects can be kept under control. Under the same conditions, all other experiments are expected to be at least about 2-3 times less competitive.

Even the sensitivity achievable with COrE lies a couple of order of magnitudes above what can be reasonably expected from a cosmic variance limited ideal experiment covering the full sky. 

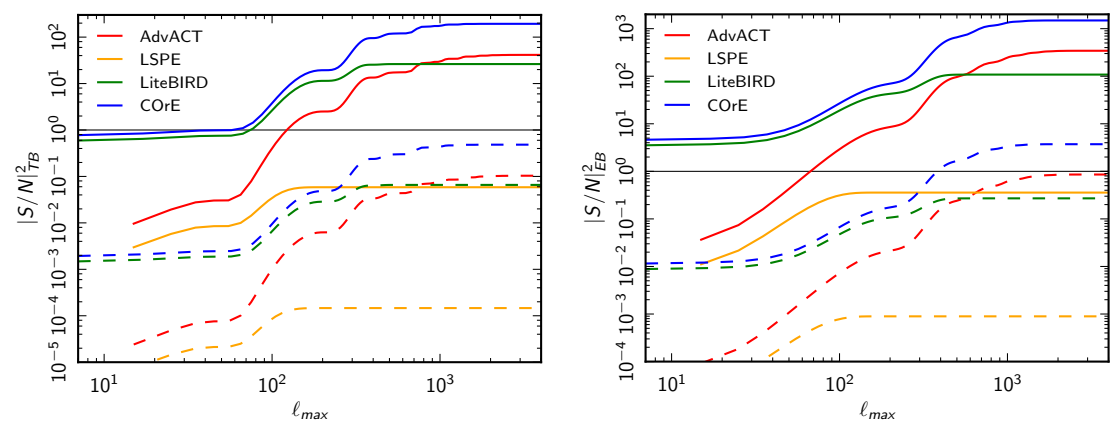

Figure 8: Left panel: Forecast of the signal-to-noise ratios for TB as defined in Eq. 25 for AdvACT (red lines), LSPE (orange lines), LiteBIRD (green lines) or COrE (blue lines). Two values of $\alpha$ are considered: $\alpha=0.1^{\circ}$ (solid lines) and $\alpha=0.005^{\circ}$ (dashed lines). Right panel: As in the left panel but for EB as defined in Eq. 26.

\section{Appendix A: Signal-to-noise ratio analysis}

In order to find the maximum multipole, $\ell_{\max }$, to be considered in our analyses, we take into account the following signal-to-noise ratio functions defined as:

$$
\begin{aligned}
& \left(\frac{S}{N}\right)_{T B}^{2}\left(\ell_{\max }\right)=\sum_{\ell=\ell_{\min }}^{\ell_{\max }}\left(\frac{C_{\ell}^{T E} \sin (2 \alpha)}{\sigma_{\ell}^{T B}}\right)^{2} \\
& \left(\frac{S}{N}\right)_{E B}^{2}\left(\ell_{\max }\right)=\sum_{\ell=\ell_{\min }}^{\ell_{\max }}\left(\frac{1}{2} \frac{\left(C_{\ell}^{E E}-C_{\ell}^{B B}\right) \sin (4 \alpha)}{\sigma_{\ell}^{E B}}\right)^{2}
\end{aligned}
$$

where $C_{\ell}^{T E}, C_{\ell}^{E E}$ and $C_{\ell}^{B B}$ are chosen to be the best fit model obtained from Planck 2015 [39] and where $\sigma_{T B}$ and $\sigma_{E B}$ are the uncertainties for the TB and EB spectra respectively.

Fig. 7 shows Eq. 25) (left panel) and 26) (right panel) for ACTpol data and considering $\alpha=0.1^{\circ}, 0.3^{\circ}, 0.6^{\circ}$ and $0.9^{\circ}$. Both left and right panel of Fig. 7 exhibit a saturation of the signal-to-noise ratio at $\ell_{\max }$ of the order of 3000. This justifies the choice of $\ell_{\max }=3000$ when we perform our analyses of ACTpol data, see Section 4 .

Similarly Fig. 8 shows Eq. 25) and 26) for future CMB experiments as listed in Table 1 considering $\alpha=0.1^{\circ}$ and $0.005^{\circ}$. It clearly displays that 
250

$\ell_{\max } \sim 600$ for LiteBIRD and $\ell_{\max } \sim 2000$ for COrE. Therefore we choose the maximum multipole accordingly in our forecasts, see Section 5 .

\section{Acknowledgments}

We acknowledge the support by ASI/INAF Agreement 2014-024-R.1 for the 255 Planck LFI Activity of Phase E2.

\section{References}

[1] Planck Collaboration, Planck 2015 results. I. Overview of products and results, A\&A 594 (2016) A1. arXiv:1502.01582

[2] BICEP2/Keck Array and Planck Collaborations, Joint Analysis of BICEP2/Keck Array and Planck Data, Physical Review Letters 114 (10) (2015) 101301.

[3] J. L. Sievers, R. A. Hlozek, M. R. Nolta, et al., The Atacama Cosmology Telescope: cosmological parameters from three seasons of data, JCAP 10 (2013) 60 .

${ }_{265}^{6}$ [4] K. K. Schaffer, T. M. Crawford, K. A. Aird, et al., The First Public Release of South Pole Telescope Data: Maps of a $95 \mathrm{deg}^{2}$ Field from 2008 Observations, APJ 743 (2011) 90.

[5] Planck Collaboration, Planck 2015 results. XI. CMB power spectra, likelihoods, and robustness of parameters, A\&A 594 (2016) A11. arXiv: 270 1507.02704 .

[6] S. W. Henderson, R. Allison, J. Austermann, et al., Advanced ACTPol Cryogenic Detector Arrays and Readout, Journal of Low Temperature Physics 184 (2016) 772-779. arXiv:1510.02809. 
[7] E. M. George, C. L. Reichardt, K. A. Aird, et al., A Measurement of Sec-

[13] J. Delabrouille, COrE + The Cosmic Origins Explorer A proposal for ESA's M4 space mission, Retrieved from the University of Minnesota Digital Conservancy, http://hdl.handle.net/11299/169642.

[14] S. M. Carroll, G. B. Field, R. Jackiw, Limits on a Lorentz- and parityviolating modification of electrodynamics, Physical Review D 41 (1990) $1231-1240$.

[15] S. M. Carroll, Quintessence and the Rest of the World: Suppressing LongRange Interactions, Physical Review Letters 81 (1998) 3067-3070.

[16] M. Giovannini, Magnetized birefringence and CMB polarization, Physical 
[17] F. Finelli, M. Galaverni, Rotation of linear polarization plane and circular polarization from cosmological pseudoscalar fields, Physical Review D 79 (6) (2009) 063002.

[18] N. Bartolo, S. Matarrese, M. Peloso, M. Shiraishi, Parity-violating CMB correlators with non-decaying statistical anisotropy, JCAP 7 (2015) 39.

[19] F. Della Valle, A. Ejlli, U. Gastaldi, G. Messineo, E. Milotti, R. Pengo, G. Ruoso, G. Zavattini, The PVLAS experiment: measuring vacuum magnetic birefringence and dichroism with a birefringent Fabry-Perot cavity, European Physical Journal C 76 (2016) 24.

[20] S. di Serego Alighieri, F. Finelli, M. Galaverni, Cosmological birefringence: an astrophysical test of fundamental physics, in: PPC 2010: IV International Workshop On The Interconnection Between Particle Physics And Cosmology, Proceedings of the conference held 12-16 July, 2010 in Torino, Italy. Online at http://www.ppc10.to.infn.it/talks.php, id.27, 2010, p. 27. arXiv:1011.4865.

[21] M. Kamionkowski, Nonuniform cosmological birefringence and active galactic nuclei, Physical Review D 82 (4) (2010) 047302.

[22] A. Lue, L. Wang, M. Kamionkowski, Cosmological Signature of New ParityViolating Interactions, Physical Review Letters 83 (1999) 1506-1509.

[23] M. Li, Y.-F. Cai, X. Wang, X. Zhang, CPT violating electrodynamics and Chern-Simons modified gravity, Physics Letters B 680 (2009) 118-124.

[24] S. di Serego Alighieri, Cosmic Polarization Rotation in view of the recent CMB experiments, ArXiv e-prints arXiv:1507.02433.

[25] J. P. Kaufman, B. G. Keating, B. R. Johnson, Precision tests of parity violation over cosmological distances, MNRAS 455 (2016) 1981-1988.

[26] C. R. Contaldi, Imaging parity-violation in the CMB, arXiv:1510.02629 
[27] A. Gruppuso, M. Gerbino, P. Natoli, L. Pagano, N. Mandolesi, D. Molinari, Constraints on cosmological birefringence from Planck and Bicep2/Keck data, JCAP 6 (2016) 1. arXiv:1509.04157.

[28] V. Gluscevic, D. Hanson, M. Kamionkowski, C. M. Hirata, First CMB constraints on direction-dependent cosmological birefringence from WMAP-7, Phys. Rev. D 86 (10).

[29] Polarbear Collaboration, P. A. R. Ade, K. Arnold, M. Atlas, C. Baccigalupi, et al., POLARBEAR constraints on cosmic birefringence and primordial magnetic fields, Phys. Rev. D 92 (12).

[30] W. Zhao, M. Li, Fluctuations of cosmological birefringence and the effect on CMB B-mode polarization, Physical Review D 89 (10) (2014) 103518.

[31] W. Zhao, M. Li, Detecting relic gravitational waves in the CMB: The contamination caused by the cosmological birefringence, Physics Letters B 737 (2014) 329-334.

[32] V. Gluscevic, M. Kamionkowski, A. Cooray, Derotation of the cosmic microwave background polarization: Full-sky formalism, Phys. Rev. D 80 (2).

[33] A. P. S. Yadav, R. Biswas, M. Su, M. Zaldarriaga, Constraining a spatially dependent rotation of the cosmic microwave background polarization, Phys. Rev. D 79 (12).

[34] E. Y. S. Wu, P. Ade, J. Bock, et al., Parity Violation Constraints Using Cosmic Microwave Background Polarization Spectra from 2006 and 2007 Observations by the QUaD Polarimeter, Physical Review Letters 102 (16) (2009) 161302.

[35] A. Gruppuso, P. Natoli, N. Mandolesi, A. De Rosa, F. Finelli, F. Paci, WMAP 7 year constraints on $\mathrm{CPT}$ violation from large angle CMB anisotropies, JCAP 2 (2012) 023. 
[36] G.-B. Zhao, Y. Wang, J.-Q. Xia, M. Li, X. Zhang, An efficient probe of the cosmological CPT violation, JCAP 7 (2015) 32.

355 [37] A. Gruppuso, G. Maggio, D. Molinari, P. Natoli, A note on the birefringence

1 angle estimation in CMB data analysis, JCAP 5 (2016) 20. arXiv:1604. 05202 .

[38] S. Naess, Private communication.

[39] Planck Collaboration, Planck 2015 results. XIII. Cosmological parameters, A\&A 594. arXiv:1502.01589

[40] M. Zaldarriaga, U. Seljak, All-sky analysis of polarization in the microwave background, Physical Review D 55 (1997) 1830-1840.

[41] M. Kamionkowski, A. Kosowsky, A. Stebbins, Statistics of cosmic microwave background polarization, Physical Review D 55 (1997) 7368-7388.

[42] H.-H. Mei, W.-T. Ni, W.-P. Pan, L. Xu, S. di Serego Alighieri, New Constraints on Cosmic Polarization Rotation from the ACTPol Cosmic Microwave Background B-mode Polarization Observation and the BICEP2 Constraint Update, APJ 805 (2015) 107. 